\title{
Females mate with males with diminished pheomelanin-based coloration in the Eurasian nuthatch Sitta europaea
}

Ismael Galván ${ }^{1, *}$ and Sol Rodríguez-Martínez ${ }^{1}$

${ }^{1}$ Department of Evolutionary Ecology, Doñana Biological Station, CSIC, Sevilla, Spain

*Correspondence. E-mail: galvan@ebd.csic.es

Running title: Female preference for diminished color expression

\section{Declarations}

Acknowledgements. We thank Javier Pérez-Tris and an anonymous reviewer for constructive comments on the manuscript, and Rafael Márquez his help with the field work and with the spectrophotometric analyses of feathers.

Funding. Funding support was received from the project CGL2015-67796-P from the Spanish Ministry of Economy, Industry and Competitiveness (MINECO). IG benefited from

a Ramón y Cajal Fellowship (RYC-2012-10237) and SRM from the grant BES-2016077112, both from MINECO.

Author contributions. Both authors contributed equally to this paper.

Conflicts of interest. Declared none.

Permits. This study received approval by the Bioethics Subcommittee of the Spanish National Research Council (CSIC) on 23th February 2015, and was conducted with the authorization \#06-04-15-227 by local authorities (Consejería de Agricultura y Pesca y Desarrollo Rural, Junta de Andalucía). 


\section{Females mate with males with diminished pheomelanin-based}

\section{2 coloration in the Eurasian nuthatch Sitta europaea}

Abstract

Sexual selection can drive the evolution of phenotypic traits because of female

preferences for exaggerated trait expression in males. Sexual selection can also lead to the evolutionary loss of traits, a process to which female preferences for diminished male trait expression are hypothesized to contribute. However, empirical evidence of female preferences for diminished male traits is virtually lacking. Eurasian nuthatches Sitta europaea provide an opportunity to test this possibility, as a chestnut flank patch produced by the pigment pheomelanin is present since the first plumage of these birds and its color is more intense in nestlings in poor condition in our study population. It has been proposed that developing birds in poor condition may increase their production of pheomelanin as a detoxifying strategy. Female nuthatches may thus prefer mating with males showing flank feathers of diminished color, as this could indicate that males experienced good conditions early in development, which can positively affect the fitness of future generations. Here we show results according with this prediction in a wild population of Eurasian nuthatches, as adult males with lighter chestnut feathers paired earlier in the season, while chestnut coloration had no effect on female mating success. Chestnut color expression was not affected by the body condition of birds, suggesting that females obtain information on the body condition in early life of their potential mates and not on their current body condition.

22 This constitutes one of the few examples of females mating with males showing 23 diminished traits and provides the only explanation so far by which this process can occur. Key-words: early life effects, female preference for diminished traits, nuthatch, plumage coloration, pheomelanin, sexual selection 


\section{Introduction}

27 Understanding variation in female preferences for male traits helps explain how

28 phenotypes evolve by sexual selection (Jennions and Petrie 1997). Some models of

29 sexual selection predict that this occurs as an endless process in which periods of female

30 preference for exaggerated phenotypic traits (e.g., large morphological or intense color

31 characters) alternate with periods of preference for diminished traits (i.e., perpetual

32 evolution; Mead and Arnold 2004). Indeed, phylogenetic comparisons between species

33 show frequent losses of sexually selected traits (Wiens et al. 2011), a phenomenon to

34 which female preferences for diminished male traits may contribute (Wiens 2001).

35 However, almost two decades after the proposal of this mechanism, the empirical

36 evidence of female preferences for diminished male traits not associated to heterospecific

37 mating avoidance or predation risk remains limited to one example already echoed by

38 Wiens (2001): a population of the house sparrow Passer domesticus in which females, in

39 contrast to females in other populations (Nakagawa et al. 2007), prefer mating with males

40 displaying smaller black bib plumage patches (Griffith et al. 1999). This may suggest that

41 the theoretical possibility of female preference for diminished male traits has not been

42 assumed by researchers, as findings in this direction may be disregarded as incoherent

43 evidence of sexual selection.

Although female preferences can drive the evolution of male traits by an arbitrary attractive-only effect (Fukamachi et al. 2009), empirical studies suggest that in most cases females obtain adaptive benefits from their choices, either directly by getting genetic pools that increase the viability of their offspring or indirectly by getting resources that help raising the offspring (e.g., Jaquiéry et al. 2010). In this regard, understanding the mechanisms that regulate the expression of male traits is essential to get insight into

50 female preferences (Folstad and Karter 1992), as these preferences are mostly

51 understood through the information that male traits honestly signal about their ability to 
52 produce the traits (Zahavi 1975). This information is in turn necessary to evaluate the

53 likelihood that female preferences for diminished male traits actually operates at any time.

Recent findings in the Eurasian nuthatch Sitta europaea provide a unique opportunity to test a possibility of female preference for a diminished male traits. These birds are sexually dichromatic regarding a chestnut (dark orange) flank plumage patch produced by the deposition of the pigment pheomelanin in feathers, whose color intensity is higher in males than in females (Fig. 1). This color patch is already present in the first plumage developed by nestlings, which are virtually identical to adults (Fig. 1). However, the color intensity of this trait depends on the physical condition, a predictor of survival prospects (Matthysen 1989), in nestlings but not in adults: nestling nuthatches in poorer body condition develop more intense flank feathers than nestlings in better condition in at least one population (Galván 2017). As the synthesis of pheomelanin and its deposition in inert integumentary structures like feathers can help removing the amino acid cysteine, which is toxic if in excess and this excess is more likely to arise during the nesting stage However, the plumage of nuthatches is sexually dichromatic as mentioned above, indicating that sexual selection operates on this trait (Heinsohn et al. 2005). The absence of condition-dependence in adult nuthatches suggests that preferences of adult females for male flank coloration might be related to information different from the current body experienced in early life by their potential mates by assessing the color intensity of their flank patches, as suggested for some morphological traits in other birds with marked sexual dimorphism (Carranza and Hidalgo de Trucios 1993). This would only be possible if 
78 plumage color expression was correlated between years in individual males, and this is

79 indeed likely, as bird color traits involved in sexual selection uses to be consistent across 80 years (Galván and Møller 2009, Gladbach et al. 2010, Chaine and Lyon 2015). This

81 information may be highly relevant for the fitness of female nuthatches, as conditions experienced in early life can have transgenerational effects (Burton and Metcalfe 2014). In birds specifically, it is known that stress experienced early during development can lead to poor body condition in the affected offspring and negative fitness-related effects in subsequent generations (Naguib et al. 2006, Goerlich et al. 2012). Therefore, female nuthatches would benefit from mating with males with a diminished expression of flank coloration, as this would be indicative of good physical condition during development (Galván 2017). We thus tested this prediction in a wild population of Eurasian nuthatches using laying date as a measure of mating success, as laying date reflects pairing date (Potti and Montalvo 1991, Johnsen et al. 1998).

\section{Material and Methods}

\section{Field methods}

The study was carried out during three consecutive breeding seasons (April-May 2015-

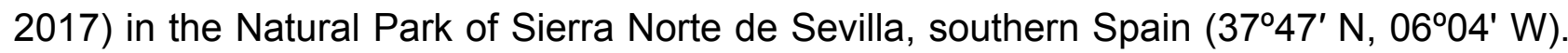
Frequent checks of wood nest boxes placed in the study area provided data on dates of clutch initiation (laying date) for all breeding pairs.

12-15 days after hatching, adults were captured and banded with numbered rings.

The adults were weighed with a portable electronic balance to the nearest $0.1 \mathrm{~g}$ and their tarsus length was measured to the nearest $0.01 \mathrm{~mm}$ with a digital calliper. Sex was determined on the basis of the color intensity of the flank plumage patch, which is darker in males (Galván 2017). We plucked 5-6 chestnut flank body feathers and stored them in the dark until reflectance measurements were made (see below). In total, 37 Eurasian 
104 nuthatches (17 males and 20 females) belonging to 27 breeding pairs were captured and 105 examined for flank coloration. Flank feathers could not be taken in one female, and body 106 mass could not be measured in other two females.

\section{Analysis of plumage color expression}

109 To analyze the color expression of flank feathers, we used an Ocean Optics Jaz 110 spectrophotometer (range 220-1000 nm) with ultraviolet (deuterium) and visible (tungsten-

111 halogen) lamps and a bifurcated 400 micrometer ber optic probe. The fiber optic probe 112 both provided illumination and obtained light reflected from the sample, with a reading 113 area of ca. $1 \mathrm{~mm}^{2}$. Feathers were mounted on a light absorbing foil sheet (Metal Velvet 114 coating, Edmund Optics, Barrington, NJ) to avoid any background reflectance. 115 Measurements were taken at a $90^{\circ}$ angle to the sample. All measurements were relative 116 to a difuse reflectance standard tablet (WS-1, Ocean Optics, Dunedin, FL), and reference 117 measurements were frequently made. An average spectrum of five readings on different 118 points of the feathers was obtained for each bird, removing the probe after each 119 measurement. Reflectance curves were determined by calculating the median of the 120 percent reflectance in $10 \mathrm{~nm}$ intervals. Spectral data was summarized as the summed 121 reflectance across the $300-700 \mathrm{~nm}$ range. As pheomelanin reflectance spectra are 122 approximately linear and spectral variation largely correspond to variation in the slope of 123 these lines (Galván and Wakamatsu 2016), we also calculated the slope of percentage 124 reflectance regressed against wavelength as an alternative measure of pheomelanin125 based color expression. In both parameters, summed reflectance and reflectance slope, 126 lower values denote darker colors and higher color intensity. Then we determined which 127 parameter was a better indicator of the pheomelanin content of feathers (see below). To 128 minimize observer bias, reflectance measurements were made by a technician blindly from 129 the aims of the study. 
Analysis of feather pheomelanin content

132 The flank feathers of 16 nuthatch nestlings from 2017 were analyzed by micro-Raman 133 spectroscopy to determine their relative content of melanins, as both pheomelanin and the 134 non-sulphurated form of melanin (eumelanin) exhibit distinctive Raman signal that can be 135 used to non-invasively identify and quantify them (Galván et al. 2013, Galván and Jorge 136 2015). We used a Thermo Fisher DXR confocal dispersive Raman microscope (Thermo 137 Fisher Scientific, Madison, WI, USA) with a point-and-shoot Raman capability of $1 \mu \mathrm{m}$ 138 spatial resolution and using a NIR excitation laser of $780 \mathrm{~nm}$. Laser power was set at 5-7 $139 \mathrm{~mW}$, integration time at $1 \mathrm{~s}$, and number of accumulations at 8 . The spectra were obtained 140 using a 50x confocal objective and a slit aperture of $50 \mu \mathrm{m}$. The system was operated with 141 Thermo Fisher OMNIC 8.1 software. Calibration and alignment of the spectrograph were 142 checked using pure polystyrene.

A total of five flank feathers were analyzed per nestling. In each feather, we 144 obtained five Raman spectra from different barbs and barbules. Pheomelanin shows a 145 distinctive Raman spectrum comprising three diagnostic bands located at 500, 1500 and $1462000 \mathrm{~cm}^{-1}$, and eumelanin shows different spectra with other diagnostic bands (Galván et 147 al. 2013, Galván and Jorge 2015, Wang et al. 2016, Polidori et al., 2017). In the nuthatch 148 feathers, we only detected Raman signal from pheomelanin, together with bands that can 149 be assigned to keratin (Hsu et al. 1976) (Fig. 2). Therefore, we used the three diagnostic 150 bands of pheomelanin and four diagnostic bands of keratin (Fig. 2a) to fit Voigt 151 deconvolution functions to the Raman curves to obtain spectral parameters derived from 152 each spectrum. An average Raman spectrum was calculated for the spectra obtained from 153 the five feathers of each nestling. In the analyses, we used the intensity (maximum value at the vertical axis) of the 155 band at $2000 \mathrm{~cm}^{-1}$ calculated from the deconvolution functions as an index of pheomelanin 
156 content (Figure 2a), as this is a good predictor of pheomelanin concentration in feathers

157 (Galván et al. 2013) and the band is located within the so-called Raman "silent region" of 158 biomolecules (i.e., $1800-2700 \mathrm{~cm}^{-1}$; Wang et al. 2016). The intensity of this band increases 159 with increasing the concentration of pheomelanin in feathers (Galván et al. 2013).

We thus regressed the slope and the summed reflectance of feathers against the 161 intensity of the $2000 \mathrm{~cm}^{-1}$ Raman band to determine which color trait better reflects the 162 pheomelanin content of feathers. The slope of reflectance spectra was significantly 163 correlated with the intensity of the $2000 \mathrm{~cm}^{-1}$ Raman band $(r=-0.51, n=16, P=0.042$; 164 Fig. 2b), while the summed reflectance was not $(r=-0.35, n=16, P=0.177$; Fig. $2 \mathrm{c})$. This 165 indicates that the slope of the regression of reflectance values against wavelength 166 decreases as the pheomelanin content of feathers increases (Fig. 2b). Although the effect 167 size is not large, its statistical significance with a sample size as low as 16 birds suggests 168 that the association might be biologically relevant. We therefore used reflectance slope as an index of pheomelanin-based color expression in the analyses.

\section{Statistical analyses}

172 We tested for a potential dependence of laying date on the flank color expression of males 173 and females separately by using general linear models (GLM). We standarized laying date 174 (mean $=0$, s.d. $=1$ ) to compare values between the three years of study. Standardized laying date values thus represent deviations from the yearly means. We also tested for an association between flank color expression and body condition, the latter being calculated

177 as the residuals of body mass regressed against tarsus length (Galván 2017). Males and 178 females were pooled in these models for body condition, and the sex of birds was added 179 as a covariate to account for the effect of sexual dichromatism. Inspections of residuals 180 from the models confirmed that normality assumptions were fulfilled. Lastly, we tested for a 
181 correlation between the color expression of males and females in 10 pairs in which we 182 could capture both sexes.

\section{Results}

185 Flank color expression explained $32.8 \%$ of variance in the laying date of male nuthatches, exerting a significant and negative effect $\left(b=-68.88, F_{1,15}=7.31, P=0.016\right)$. This means that males displaying lighter chestnut plumage patches paired earlier in the season (Fig. 3). In contrast, flank color expression in females only explained $21.8 \%$ of variance in their laying date, and the effect was not significant $\left(b=15.98, F_{1,17}=1.64, P=0.218 ;\right.$ Fig. 3$)$.

In the model testing for an effect of body condition on flank color expression, neither

191 the interaction between body condition and $\operatorname{sex}\left(F_{1,30}=0.16, P=0.688\right)$ nor body condition $192\left(F_{1,31}=0.88, P=0.356\right)$ had a significant effect. Only the sex of birds had a significant 193 effect $\left(F_{1,34}=10.7, P=0.002\right)$, indicating darker flank plumage patches in males (mean \pm s.e.: $0.024 \pm 0.003)$ than in females $(0.038 \pm 0.003)$.

The correlation between the flank color expression of paired males and females was not significant $(r=-0.40, n=10, P=0.250)$. This suggests that the flank color of males was unrelated to that of their mates.

\section{Discussion}

200 Our results suggest that female Eurasian nuthatches tend to mate with males with a 201 diminished expression of chestnut flank coloration, as predicted on the basis that the 202 intensity of chestnut coloration is higher in nestling nuthatches in poor body condition 203 (Galván 2017). In contrast, the flank coloration of female nuthatches does not affect their mating behavior, suggesting the existence of preferences of females to males but not the opposite. Accordingly, there was no assortative mating regarding flank color, as the color 
207 traits that are involved in sexual selection tend to be consistent across years (Galván and 208 Møller 2009, Chaine and Lyon 2015). Thus, and although we could not test this in 209 nuthatches, it is likely that the intensity of flank color expression in male nuthatches is 210 correlated across their multiple plumage molts throughout their lifespans, and therefore 211 females could obtain information on the conditions experienced during early life by 212 assessing the flank color expression of their potential mates. As birds reared under 213 stressful conditions that lead to poor body condition (Krause et al. 2017) can exert a 214 negative effect on the fitness of birds of subsequent generations (Naguib et al. 2006), the 215 tendency of female nuthatches to mate with males with lighter flank coloration may be 216 adaptive, as they may potentially avoid mating with males that provide a negative effect on 217 their offspring. These effects can be exerted through the heritability of epigenetic 218 mechanisms that influence the expression of genes that regulate stress responses 219 (Goerlich et al. 2012). As already noted by Galván (2017), the expression of the chestnut flank coloration 221 does not depend on the current body condition of adult nuthatches, and thus, females 222 cannot obtain information on the current body condition of their potential mates by 223 assessing their flank coloration. This may thus be an exception to the observation that 224 sexually selected traits often, but not always, show heightened condition-dependence and 225 thus signal the capacity to afford the costs of producing the traits (Cotton et al. 2004). In 226 this case, the reason for the absence of condition-dependence in adult nuthatches may be 227 the proposed detoxifying function of pheomelanin, the pigment that produces the flank 228 coloration and whose synthesis increases in developing nuthatches in poor body condition 229 (Galván 2017). Therefore, in our study population the chestnut flank coloration is not a trait 230 costly to produce whose expression is limited to individuals of high genotypic quality as 231 predicted by the handicap principle (Zahavi 1975), but is more intensely produced by 232 individuals in poor condition probably as an adaptive detoxifying strategy. This is in 
233 accordance with recent proposals of alternatives to the handicap principle that are based

234 on the knowledge of the physiological mechanisms regulating trait expression (Számadó and Penn 2015, Galván and Alonso-Alvarez 2017). This is also in accordance with the suggestion that the chestnut flank coloration of adult nuthatches is a developmental consequence of natural selection operating in nestlings, while sexual dichromatism in the chestnut flank coloration of nestling nuthatches is a developmental consequence of sexual selection operating in adults (Galván 2017).

It must be noted that, in other passerine bird (the barn swallow Hirundo rustica), the expression of pheomelanin-based plumage coloration is positively related to body condition in nestlings (Arai et al. 2017) and to annual survival rate in adults (Galván and Møller 2013), and, accordingly, females show a preference for males with intense plumage 244 coloration in at least some populations (Scordato and Safran 2014). Between-species 245 comparisons indicate that the production of large amounts of pheomelanin to pigment plumage constrains physiological processes that require antioxidant protection by glutathione (GSH), the most important intracellular antioxidant, such as the development of large brains (Galván and Møller 2011) or performance under exposure to ionizing radiation (Galván et al. 2014). This is because the sulfhydryl group of cysteine, a 250 constituent amino acid of $\mathrm{GSH}$, enters the pheomelanin synthesis pathway, thus 251 pheomelanin production reduces the avalability of cysteine for GSH synthesis and this can 252 cause chronic oxidative stress (Napolitano et al. 2014). However, only an adaptive benefit 253 of pheomelanin can explain its evolution, and such benefit may be the detoxifying capacity 254 of pheomelanin by helping to avoid excess cysteine, which is toxic (Galván et al. 2012). 255 Therefore, it is environmental conditions what determine whether pheomelanin production is physiologically advantageous or detrimental (Galván et al. 2012), which may explain 257 differences in the condition-dependence and signaling content of pheomelanin-based coloration between species. In fact, we have evidence that the expression of color of flank 
259 feathers in Eurasian nuthatch nestlings reared in a population different from the population 260 of the present study, with different levels of environmental oxidative stress, shows the 261 reversed pattern to that found here (i.e., color intensity is positively associated to body 262 condition; Galván and Sanz, unpublished results). Future studies should thus consider 263 environmental gradients of oxidative stress when explaining the signaling content of 264 pheomelanin-based coloration.

To our knowledge, our study is the only finding suggesting that females may show preferences for a diminished male trait not associated to heterospecific mating avoidance or predation risk since the results provided by Griffith et al. (1999) in a population of house sparrows. This possibility, however, theoretically exists in certain models of sexual selection (Mead and Arnold 2004) and may actually be responsible for the evolutionary loss of sexually selected traits observed in some clades (Wiens 2001). The potential 271 detoxifying function of pheomelanin-based pigmentation provides a mechanism by which 272 females tend to mate with males with a diminished expression of pheomelanin-based 273 coloration as we found here, thus representing the first mechanistic justification for such 274 female mating behavior. Indeed, the evolution of signals can be understood only by 275 considering the physiological mechanisms that regulate their expression (Galván and Alonso-Alvarez 2017).

The question now arises why the flank plumage patch of nuthatches does not become unpigmented, if females tend to mate with males with lighter flanks and this potentially provide them with higher fitness benefits. The answer might be in the stressful 280 factors that create differences in the body condition of nuthatches during development. 281 Nuthatch populations may differ in the expression of flank coloration, which would suggest 282 that populations may differ in the intensity of stressful factors and maybe also in the 283 intensity of sexual selection (Galván and Sanz, unpublished results). Thus, similar to the 284 black bib of male house sparrows (Griffith et al. 1999, Nakagawa et al. 2007), preferences 
285 of female nuthatches for the flank coloration of males may differ between populations with 286 different intensity of stressful factors affecting body condition in early life. This should be 287 investigated by future studies, as it may help understanding seemingly paradoxical 288 findings in sexual selection and obtaining a more complete view of the influence of this 289 process in phenotypic evolution.

\section{References}

292 Arai, E., Hasegawa, M., Makino, T., Hagino, A., Sakai, Y., Ohtsuki, H., Wakamatsu, K. and 293 Kawata, M. 2017. Physiological conditions and genetic controls of phaeomelanin pigmentation in nestling barn swallows. Behavioral Ecology 28, 706-716.

Burton, T. and Metcalfe, N.B. 2014. Can environmental conditions experienced in early life influence future generations? Proceedings of the Royal Society B 281, 20140311.

Carranza, J. and Hidalgo de Trucios, S.J. 1993. Condition-dependence and sex traits in the male great bustard. Ethology 94, 187-200.

Chaine, A.S. and Lyon, B.E. 2015. Signal architecture: temporal variability and individual consistency of multiple sexually selected signals. Functional Ecology 29, 11781188.

Cotton, S., Fowler, K. and Pomiankowski, A. 2004. Do sexual ornaments demonstrate heightened condition-dependent expression as predicted by the handicap hypothesis? Proceedings of the Royal Society of London, Series B 271, 771-783.

Folstad, I. and Karter, A.J. 1992. Parasites, bright males, and the immunocompetence handicap. American Naturalist 139, 603-622.

Fukamachi, S., Kinoshita, M., Aizawa, K., Oda, S., Meyer, A. and Mitani, H. 2009. Dual control by a single gene of secondary sexual characters and mating preferences in medaka. BMC Biology 7, 64. 
310 Galván, I. 2017. Condition-dependence of pheomelanin-based coloration in nuthatches Sitta europaea suggests a detoxifying function: implications for the evolution of juvenile plumage patterns. Scientific Reports 7, 9138.

Galván, I. and Møller, A.P. 2009. Different roles of natural and sexual selection on senescence of plumage colour in the barn swallow. Functional Ecology 23, 302309.

Galván, I. and Møller, A.P. 2011. Brain size and the expression of pheomelanin-based colour in birds. Journal of Evolutionary Biology 24, 999-1006.

Galván, I. and Møller, A.P. 2013. Pheomelanin-based plumage coloration predicts survival rates in birds. Physiological and Biochemical Zoology 86, 184-192.

Galván, I. and Jorge, A. 2015. Dispersive Raman spectroscopy allows the identification and quantification of melanin types. Ecology and Evolution 5, 1425-1431.

Galván, I. and Wakamatsu, K. 2016. Color measurement of the animal integument predicts the content of specific melanin forms. RSC Advances 6, 79135-79142.

Galván, I. and Alonso-Alvarez, C. 2017. Individual quality via sensitivity to cysteine availability in a melanin-based honest signalling system. Journal of Experimental Biology 220, 2825-2833.

Galván, I., Ghanem, G. and Møller, A.P. 2012. Has removal of excess cysteine led to the evolution of pheomelanin? BioEssays 34, 565-568.

Galván, I., Jorge, A., Ito, K., Tabuchi, K., Solano, F. and Wakamatsu, K. 2013. Raman spectroscopy as a non-invasive technique for the quantification of melanins in feathers and hairs. Pigment Cell \& Melanoma Research 26, 917-923.

Galván, I., Bonisoli-Alquati, A., Jenkinson, S., Ghanem, G., Wakamatsu, K., Mousseau, T. A. and Møller, A.P. 2014. Chronic exposure to low-dose radiation at Chernobyl favours adaptation to oxidative stress in birds. Functional Ecology 28, 1387-1403. 
335 Gladbach, A., Gladbach, D.J., Kempenaers, B. and Quillfeldt, P. 2010. Female-specific colouration, carotenoids and reproductive investment in a dichromatic species, the upland goose Chloephaga picta leucoptera. Behavioral Ecology and Sociobiology 64, 1779-1789.

Goerlich, V.C., Nätt, D., Elfwing, M., Macdonald, B. and Jensen, P. 2012. Transgenerational effects of early experience on behavioral, hormonal and gene expression responses to acute stress in the precocial chicken. Hormones and Behavior 61, 711-718.

Griffith, S.C., Owens, I.P. and Burke, T. 1999. Female choice and annual reproductive success favour less-ornamented male house sparrows. Proceedings of the Royal Society of London B 266, 765-770.

Heinsohn, R., Legge, S. and Endler, J.A. 2005. Extreme reversed sexual dichromatism in a bird without sex role reversal. Science 309, 617-619.

Hsu, S.L., Moore, W.H. and Krimm, S. 1976. Vibrational spectrum of the unordered polypeptide chain: a Raman study of feather keratin. Biopolymers 15, 1513-1528.

Jaquiéry, J., Broquet, T., Aguilar, C., Evanno, G. and Perrin, N. 2010. Good genes drive female choice for mating partners in the lek-breeding European treefrog. Evolution 64, 108-115.

Jennions, M.D. and Petrie, M. 1997. Variation in mate choice and mating preferences: a review of causes and consequences. Biological Reviews 72, 283-327.

Johnsen, A., Andersson, S., Örnborg, J. and Lifjeld, J.T. 1998. Ultraviolet plumage ornamentation affects social mate choice and sperm competition in bluethroats (Aves: Luscinia s. svecica): a field experiment. Proceedings of the Royal Society of London B 265, 1313-1318. 
359 Krause, E.T., Krüger, O. and Pogány, Á. 2017. Zebra finch nestlings, rather than parents, suffer from raising broods under low nutritional conditions. Behavioral Ecology and Sociobiology 71, 152.

Matthysen, E. 1989. Territorial and nonterritorial settling in juvenile Eurasian nuthatches (Sitta europaea L.) in summer. Auk 106, 560-567.

Mead, L.S. and Arnold, S.J. 2004. Quantitative genetic models of sexual selection. Trends in Ecology and Evolution 19, 264-271.

Naguib, M., Nemitz, A. and Gil, D. 2006. Maternal developmental stress reduces reproductive success of female offspring in zebra finches. Proceedings of the Royal Society of London B 273, 1901-1905.

Számadó, S. and Penn, D.J. 2015. Why does costly signalling evolve? Challenges with testing the handicap hypothesis. Animal Behaviour 110, e9-e12. 
383 Wang, H., Osseiran, S., Igras, V., Nichols, A.J., Roider, E.M., Pruessner, J., Tsao, H., Fisher, D.E. and Evans, C.L. 2016. In vivo coherent Raman imaging of the melanomagenesis-associated pigment pheomelanin. Scientific Reports 6, 37986.

386 Wiens, J.J. 2001. Widespread loss of sexually selected traits: how the peacock lost its spots. Trends in Ecology and Evolution 16, 517-523.

388 Wiens, J.J., Sparreboom, M. and Arntzen, J.W. 2011. Crest evolution in newts: implications for reconstruction methods, sexual selection, phenotypic plasticity and the origin of novelties. Journal of Evolutionary Biology 24, 2073-2086

391 Zahavi, A. 1975. Mate selection-a selection for a handicap. Journal of Theoretical 
407

408

409

410 flank feathers (credits: Ismael Galván).

411

412 Fig. 2. a: Raman spectra of flank covert feathers (shown in the insert photograph) of 16

413 Eurasian nuthatch nestlings. Each color corresponds to a different bird. The spectra show 414 the three diagnostic Raman bands of pheomelanin at about 500, 1500 and $2000 \mathrm{~cm}^{-1}$. The 415 intensity of the band at $2000 \mathrm{~cm}^{-1}$ (marked with a red arrow) was used as an index of 416 pheomelanin concentration in feathers. Other bands at about 1350, 1430, 1600 and 1700 $417 \mathrm{~cm}^{-1}$, corresponding to keratin, were also used to deconvolute Raman spectra. b: 418 relationship between pheomelanin concentration and the summed percentage of 419 reflectance across the $300-700 \mathrm{~nm}$ in the same feathers. The line is the best-fit line. c: 420 relationship between pheomelanin concentration and the slope of reflectance spectra of 421 the same feathers. The line is the best-fit line.

422

423 Fig. 3. Relationship between laying date (standardized to reflect deviations from yearly 424 means) and color expression level of chestnut flank feathers (measured as summed 425 reflectance in the $300-700 \mathrm{~nm}$ spectral range) in male (red symbols and line) and female 426 (black symbols and line) Eurasian nuthatches. Lower reflectance levels indicate darker 427 (i.e., more intense) coloration. Lines are best-fit lines. 

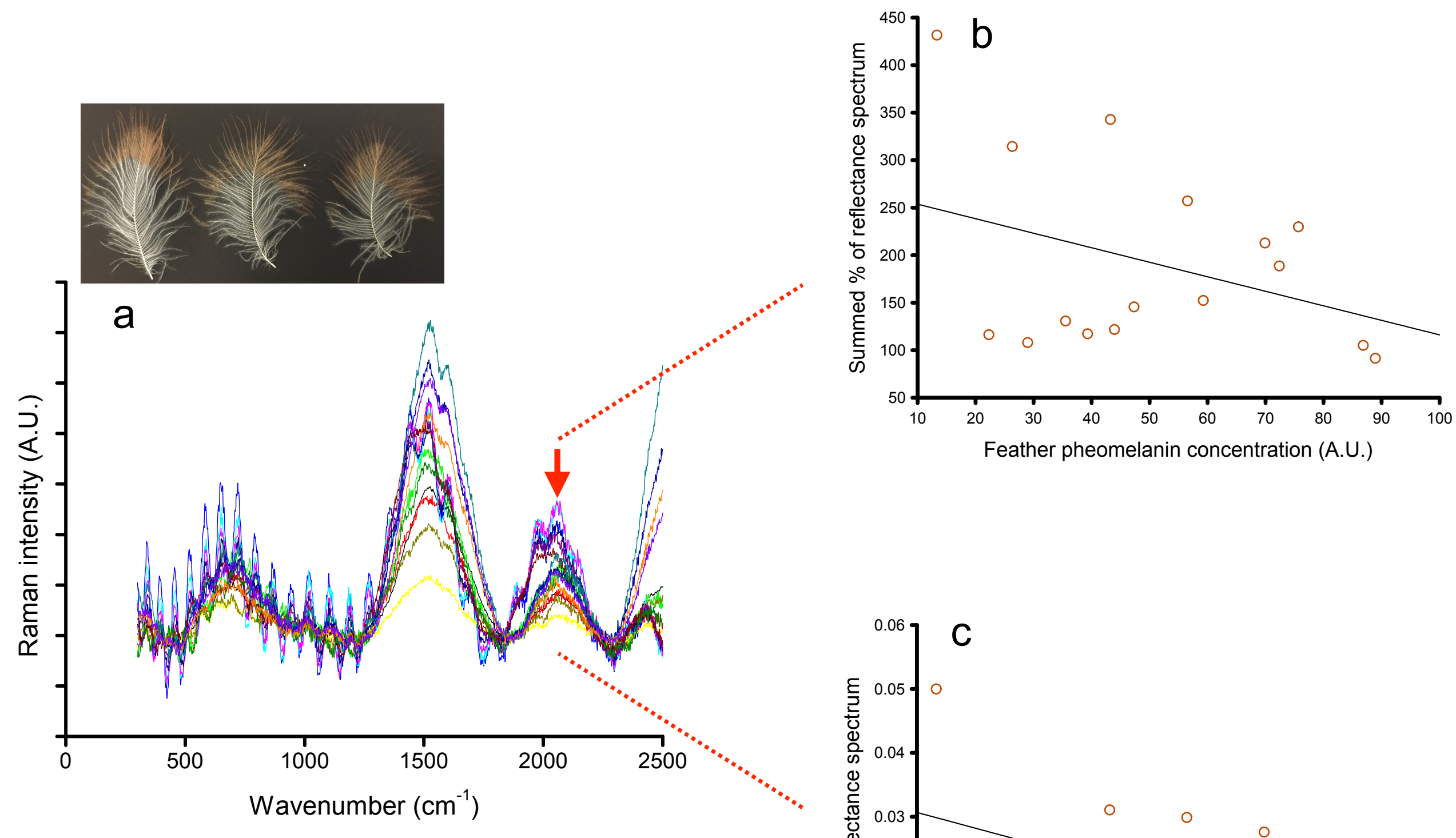

Figure 2

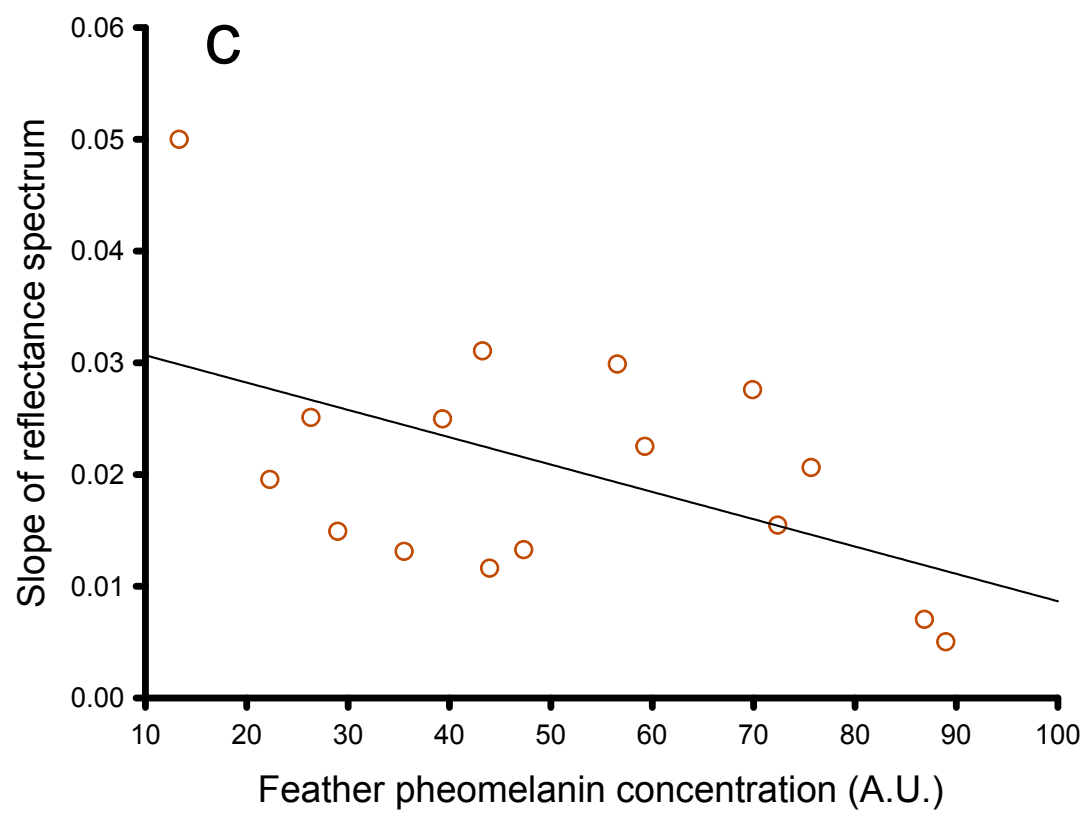




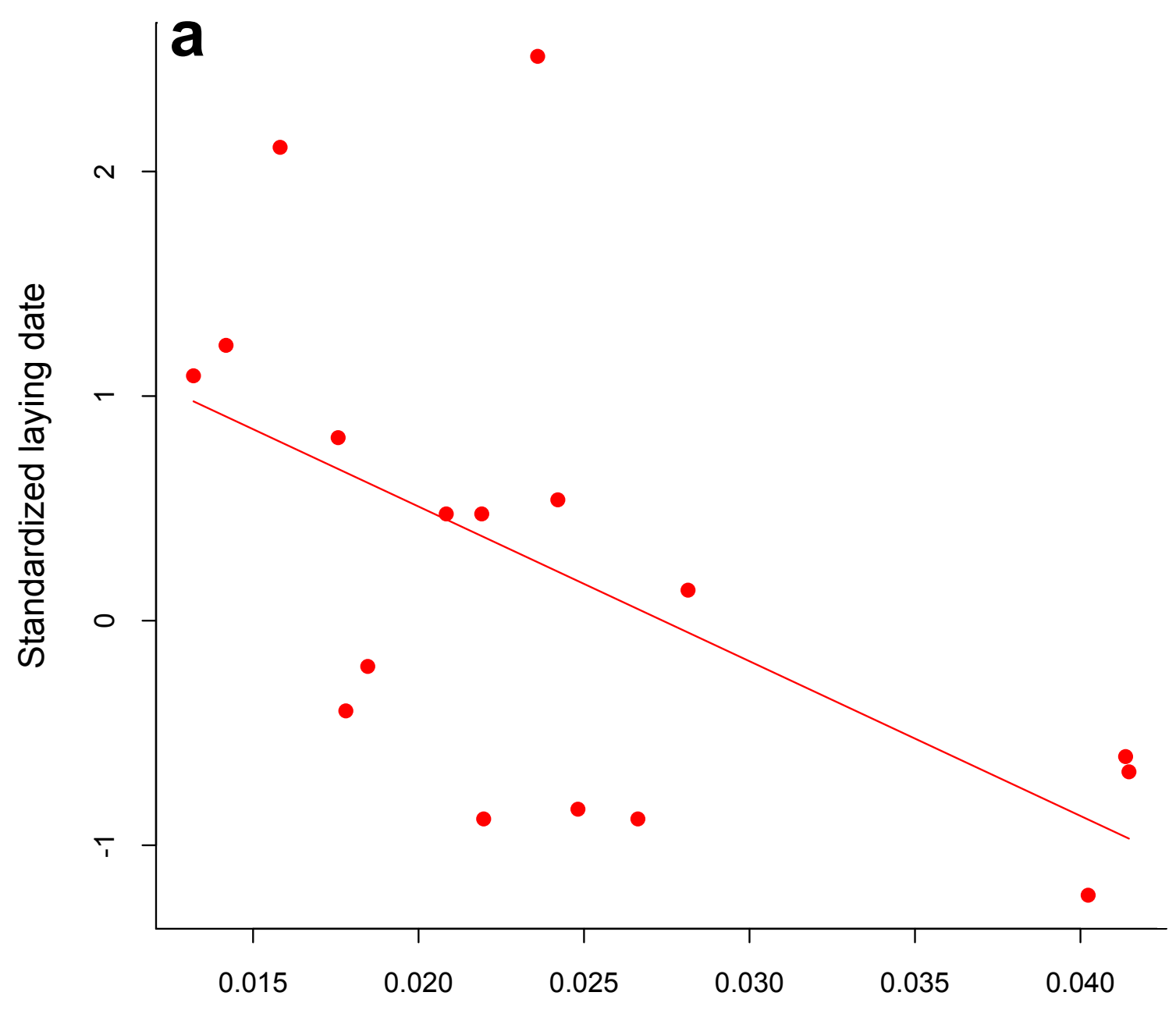

Reflectance slope of chestnut flank feathers

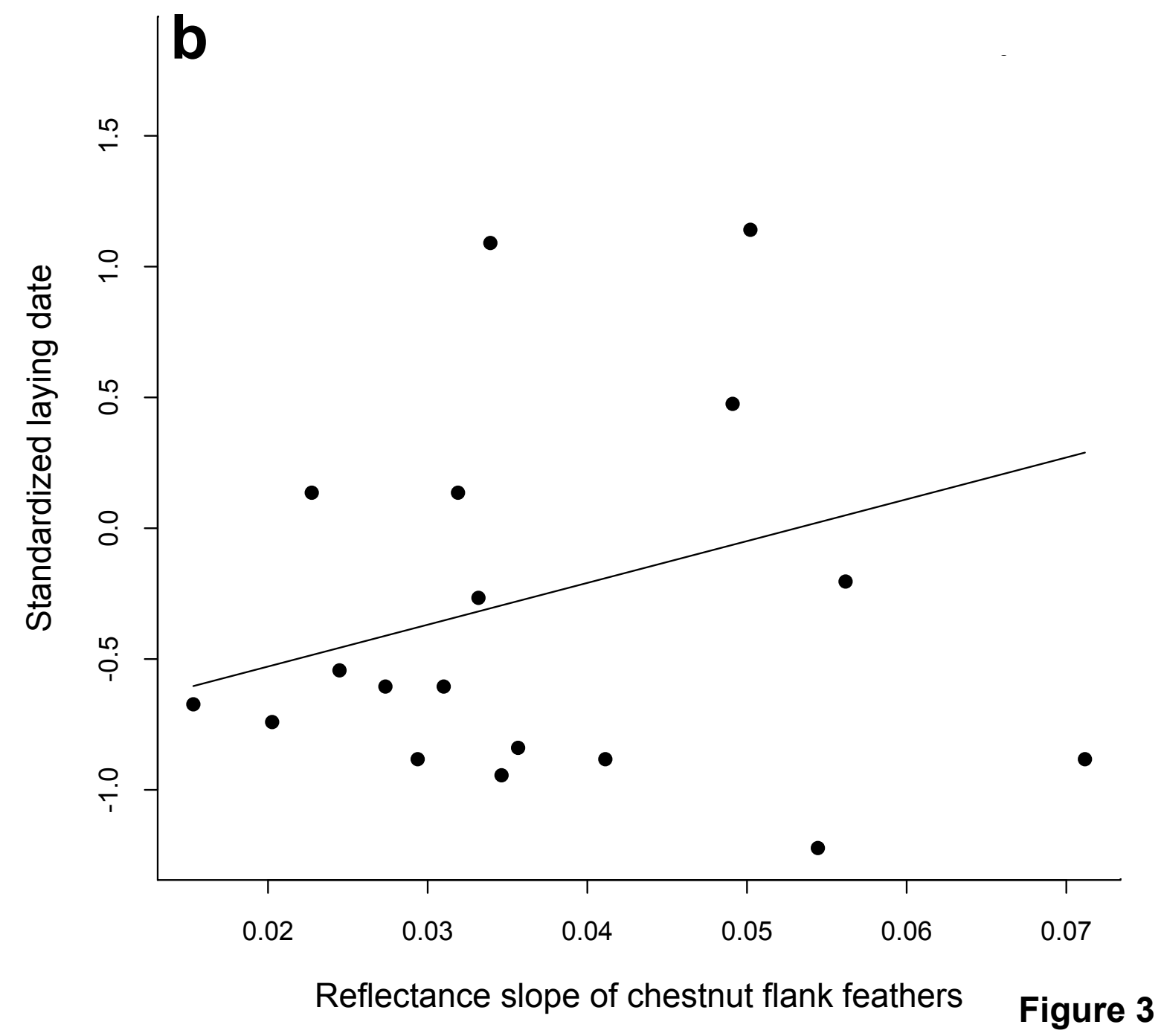

